sity) has used the methods of polymer chain statistics in assessments of DNA flexibility dependence on chain length. Her results suggest that very small changes in just a few conformational parameters of individual residues lead naturally to very considerable flexibility in the polymer as a whole.

These contributions signify some of the more promising directions in which nucleic acid structural studies are going. All of these, however 'biological' the nucleic acid environment may be in an experimental situation can only provide in vitro data. One must look in the future to techniques such as those involving NMR described by S. S. Danyluk (Argonne National Laboratory), for information from intact biological systems.

\section{Scrambling of Rydberg states by thermal radiation}

\section{from Peter Knight}

All atomic systems are immersed in a bath of blackbody radiation, a background thermal field characterised by the ambient temperature of the body. For the most part physicists interested in radiative transitions of excited atoms just ignore the existence of this field, principally because the number of blackbody photons with a frequency close to the atomic transition under study is vanishingly small, so that stimulated processes due to background thermal fields are negligible. Nevertheless, if the transition frequency is small enough, the Planck distribution of thermal photon numbers ensures that stimulated emission and absorption will begin to take over from purely spontaneous radiative decay.

Recent experiments by Gallagher and co-workers at the Stanford Research Institute have indicated that transitions between very highly excited Rydberg states are sensitive to background thermal fields. At the transition frequencies that interest them, the thermal photon occupation number at room temperature can be as high as 10 . The stimulated absorption and emission of this background radiation rapidly redistributes an initially excited state population among its neighbours and considerably shortens the lifetime of some states. Consequently it would seem quite difficult to observe the decay of a

Peter Knight is SRC Advanced Fellow at Royal Holloway College, University of London. single isolated Rydberg state in these circumstances: after a very short time a distribution of states rather than a single state exists.

In their first paper, Gallagher and Cooke (Phys. Rev. Lett. 42, 835; 1979) report the observation of the shortening of the sodium radiative np lifetimes by a factor of 3 by $300 \mathrm{~K}$ blackbody radiation induced emission and absorption. They measured the effective lifetime of the $17 p$ and $18 p$ states of sodium produced by sequential excitation by two dye laser pulses driving the $3 \mathrm{~s} \rightarrow 3 \mathrm{p} \rightarrow 18 \mathrm{p}$ or $17 \mathrm{p}$ transitions. These states can be detected by field ionisation by a d.c. field, with a pulsed technique which allows them to monitor the time-evolution of the excited state population. Their results indicate that the lifetimes are a factor of three shorter than the calculated $T=0 \mathrm{~K}$ lifetimes, in agreement with their fairly simple analysis. They believe discrepancies between theory and experiment for transitions observed by other workers can be explained by thermal background effects. They also calculate that radiative redistribution of population among neighbouring states of different $l$-quantum number due to the thermal field can be as important as collisional redistribution. This could well have astrophysical implications. Gallagher and Cooke also calculate the a.c. Stark frequency shifts produced by the thermal field. These had, in fact, been investigated theoretically some years ago by Barton (Phys. Rev. A5, 468; 1972 ) and by Knight (J. Phys. A5, $417 ; 1972)$, and the results of Gallagher and Cooke are in agreement with those earlier quantum electrodynamic calculations. The thermal field shifts all the close-lying Rydberg states upwards in energy by an equal amount of the order of kilohertz. Lowlying states are shifted by a much smaller amount according to the theoretical work of Barton and Knight. So photoabsorption from a low-lying state to a Rydberg state may well exhibit temperature-dependent shifts, although these have yet to be seen. In a further paper, Gallagher and Cooke discuss the use of Rydberg atoms as a detector of $300 \mathrm{~K}$ blackbody radiation (Appl. Phys. Lett. 34, 369; 1979), hinging on the sensitivity of field-ionisation as a monitor of highly excited state populations. They suggest that temperatures of less than $25 \mathrm{~K}$ could be detected by Rydberg-atom systems.

It is interesting to the theorist that the atom immersed in a radiation field is quite different from an isolated atom. The radiation bathing the atom also dresses it, so that what started as an isolated state is scrambled into a distribution of nearby states with transition frequencies determined at least in part by the local environment. Hot atoms aren't just moving faster than cold atoms (and therefore the ensemble emitting a wider Doppler-broadened line): each hot atom is distinctly different from a cold atom in its radiative properties.

\section{Proteins of iron metabolism}

from Pauline Harrison and E. R. Huehns

THE main topics discussed at the recent, fourth International Conference on Proteins of Iron Metabolism*-transferrin, the iron transport protein, and ferritin, the iron storage protein-are both in their own way still subject to considerable controversy. Transferrin has long been known to have two ironbinding sites, but whether these are identical structurally and, if different whether this has physiological importance, as Fletcher and Huehns proposed (Nature 218, 1211; 1968), has been argued about at the previous three meetings.

B. Gorinsky (Birkbeck College, London) presented a ' $6 \AA$ resolution model' of rabbit transferrin while J. E. Abola from Pittsburgh had studied hen ovotransferrin. The rabbit molecule consists of two lobes which can be described approximately by ellipsoids. The longest axes of these ellipsoids are inclined at about $30^{\circ}$ to one another. The maximum overall dimensions of the molecule are approximately $100 \times$ $50 \times 40 \AA$. Both lobes exhibit clefts in the vicinity of the join. In one lobe the cleft is close to a column of strong electron density which could be $\alpha$ helix, and on the opposite side of this lobe there is a strong feature which at higher resolution may prove to be $\beta$ sheet. From other work it can be concluded that there is one iron-binding site on each domain and that the Nterminal binding site can be distinguished from the C-terminal one. Several investigators (J. Williams, Bristol; P. Aisen, New York; H. G. Van Eijk, Rotterdam) utilised electrophoresis in $6 \mathrm{M}$ urea, as described by Seal and Mackay (Biochim. biophys. Acta 453, 250; 1976), to separate the four species of transferrin-apotransferrin, diferric transferrin, and the two species of monoferric transferrin, one with iron on the $\mathrm{N}$-terminal site only and the other with iron on the C-terminal site only-and to measure their distribution after the addition of iron to apotransferrin in vitro and in

*Held in Davos on 17-21 April 1979. 